\title{
INVERSES OF MEASURES ON A CLASS OF DISCRETE GROUPS
}

\author{
C. KARANIKAS \\ Department of Mathematics \\ Faculty of Sclence \\ Aristotle Universlty of Thessaloniki \\ 54006 Thessalonik1, Greece \\ (Recelved February 12, 1990)
}

ABSTRACT. We examine a class of groups $G$, having a certain growth condition. We given an estimate for the norm of the inverse of an element in $l_{1}(G)$ in terms of the spectral radius and the cardinality of the support.

KEY WORDS AND PHRASES. Discrete groups, spectral radius and measure algebra. 1980 AMS SUBJECT CLASSIFICATION CODES. 43A05, 43A10, 22 D05.

1. INTRODUC'TION.

Throughout this work $G$ is a discrete group and $M(G)$ is the usual measure algebra on $G$ : we write $\delta$ for the unit of $M(G), \mu^{*} v$ for the (convolution) product of two measures $\mu, \quad v \varepsilon M(G)$.

The purpose of this paper is to investigate the following problems: Let $\mu \varepsilon M(G)$ have finite support and the (convolution) inverse of $(\delta-\mu)$ exist in $M(G)$. Is it possible to estimate the norm $\left\|(\delta-\mu)^{-1}\right\|$ of $i t$ ?

One can easily realize that this problem becomes more interesting in the "limit" case, where the support of $\mu$ is infinite. One also can realize the connection of this with the general problem of the invertibility in $M(G)$; namely the characterization of the class of Hermitian groups $G$ (see [1]) or the equality of different norms and spectrums in $M(G)$ (see [2], [3] and [4]).

Our work here can be separated into two parts. In the first part we consider the class of groups $A$ and we give an estimate of $\left\|(\delta-\mu)^{-1}\right\|$ and of $\|\exp \mu\|$ in terms of the spectral radius $r(\mu)$ and of the cardinality of the support of $\mu$.

In the second part we examine the relation of the class $A$, with the class of nilpotent groups and [FC] groups (groups having finite conjugacy class) (see [1]). We show that nilpotent groups are A-groups and that the class $A$ is closed under finite extention. We should note that be Gromov's well known result, any finitely generated group $G$ with polynomial growth is a finite extention of a nilpotent group, and so it is an A-group. It is remarkable to note that all known Hermitian groups, as they are 
referred to in [1], are A-groups. We shall complete this introduction with sume definitions and notations.

We say that $G$ is an A-group if there is a map $k: J \rightarrow N$, where $J$ is the set of all finte subsers of $G$, and $a \lambda \varepsilon \quad N$ such that for any $F \varepsilon J$

$$
\#\left(F^{n}\right)<\left(\begin{array}{c}
k(F)+n-1 \\
n
\end{array}\right)^{\lambda}, n \in N
$$

where $F^{n}=F F \ldots F$ (n-times) and $\# F$ is the candinality of $E$.

An $n$-word in the elements of $F$ is any (reduced) word of length $n$. We denote by $\left(F^{n}\right)^{\prime}$ a collection of $n$-words in the elements of $F$ which as a subset of $G$ consists of all distinct elements of $F^{n}$. Finally we shall denote by $\mu^{n}$ the convolution product $\mu^{*} \mu \ldots *^{*} \mu$ (n-times); the spectral radius $r(\mu)$ of $\mu$ is the 1 imit $\underset{n \rightarrow \infty}{ }|| \mu^{n} \|^{1 / n}$.

2. NORMS OF CERTAIN INVERTIBLE MEASURES.

We are going to show the following:

THEOREM 2.1. Let $G$ be an A-group and let $\mu$ be an element in $M(G)$, with finite support $F$ and $r(\mu)<1$. Then $\delta-\mu$ is invertible such that

$$
\left\|(\delta-\mu)^{-1}\right\|<(1-r(\mu))^{-K(F)^{\lambda}}
$$

where $k(F)$ and $\lambda_{n}$ are constants determined from the A-group structure. Furthermore

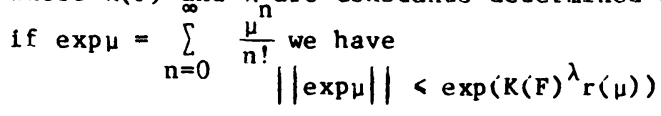

PROOF. First we observe that for any $n \varepsilon N$

$$
\begin{aligned}
\left\|\mu^{\mathrm{n}}\right\| & =\sum_{x \in G}\left|\mu^{\mathrm{n}}(\mathrm{x})\right| \\
& <\#\left(F^{\mathrm{n}}\right)^{1 / 2}\left\|\mu^{\mathrm{n}}\right\|_{2} \\
& <\#\left(F^{\mathrm{n}}\right)^{1 / 2} \sup \left\|\mu^{\mathrm{n}} \mathrm{g}\right\|_{2},\left(\mathrm{~g} \in \mathrm{l}_{2}(\mathrm{G}),\|\mathrm{g}\|_{2}=1\right) \\
& <\#\left(\mathrm{~F}^{\mathrm{n}}\right)^{1 / 2} \rho(\mu)^{\mathrm{n}}
\end{aligned}
$$

where $\|\cdot\|_{2}$ is the norm in $l_{2}(G)$ and $\rho(\mu)$ is the norm of the left regular representation, $1 . e$. the norm of the operator $\mu: 1_{2}(G)+1_{2}(G): f \rightarrow \mu^{\star} f$. Since $\rho(\mu)<r(\mu)$ we have

$$
\left\|\mu^{n}\right\|<\#\left(F^{n}\right)^{1 / 2} r(\mu)^{n}
$$

Now by (2.3) 


$$
\begin{aligned}
\left\|\delta+\mu+\mu^{2}+\ldots\right\|<1+\#(F)^{1 / 2} r(\mu)+ & \\
& +\#\left(F^{2}\right)^{1 / 2} r(\mu)^{2}+\ldots \\
& <\sum_{n=0}^{\infty}(\stackrel{k(F)+n-1}{n})^{\lambda} r(\mu)^{n}
\end{aligned}
$$

Now let $K=k(F)$ we see that $\left(\begin{array}{c}K+n-1 \\ n\end{array}\right)^{\lambda}<\left(\begin{array}{c}k^{\lambda}+n-1 \\ n\end{array}\right)$. In fact,

$$
\begin{aligned}
&\left(\begin{array}{c}
K+n-1 \\
n
\end{array}\right)^{\lambda}=\prod_{j=1}^{n}\left(\frac{K-1}{j}+1\right)^{\lambda} \\
&=\prod_{j=1}^{n}\left(1+\sum_{m=1}^{\lambda}\left(\begin{array}{l}
\lambda \\
m
\end{array}\right)\left(\frac{K-1}{j}\right)^{m}\right) \\
&<\prod_{j=1}^{n}\left(\frac{1}{j}[(K-1)+1]^{\lambda}+\frac{j-1}{j}\right) \\
&<\prod_{j=1}^{n}\left(\frac{k^{\lambda}-1}{j}+1\right) \\
&<\left(k^{\lambda+n-1}\right) \\
& n
\end{aligned}
$$

Thus

$$
\left.\| \delta+\mu+\mu^{2}+\ldots||<\sum_{n=0}^{\infty}\left({ }^{k(F)}\right)^{\lambda}+n-1\right) r(\mu)^{n}
$$

and since $r(\mu)<1$, by the binomial formula, $(\delta-\mu)^{-1}$ exists and we obtain (2.1).

To see (2.2) we observe that since $k>1$ and $j>1,\left(\frac{k-1}{j}+1\right)<k$ and

$$
\begin{aligned}
\left(\begin{array}{c}
k(F)+n-1 \\
n
\end{array}\right) & <\prod_{j=1}^{n}\left(\frac{k(F)-1}{j}+1\right)^{\lambda} \\
& <k(F)^{n \lambda}
\end{aligned}
$$

Thus by $(2.3)$ and $(2.4)$

$$
\begin{aligned}
\|\exp \mu\| & <\sum_{n=0}^{\infty} \frac{\#\left(F^{n}\right)}{n !} r(\mu)^{n} \\
& <\sum_{n=0}^{\infty} \frac{1}{n !}\left(\begin{array}{c}
k(F)+n-1 \\
n
\end{array}\right)^{\lambda} r(\mu)^{n} \\
& <\sum_{n=0}^{\infty} \frac{1}{n !}\left(K(F)^{\lambda} r(\mu)\right)^{n} \\
& =\exp \left(K(F)^{\lambda} r(\mu)\right) .
\end{aligned}
$$

3. THE CLASS OF GROUPS A.

In this section, we show that [FC] groups and nilpotent groups are A-groups. We also show that the class $A$ is closed under finite extention.

First we examine the growth of [FC] groups. 
PROPOS[TION 3.1. Let. $G$ be an [FC] group and $F$ be a finl:e subset. of $G$. Then

$$
\#\left(F^{r}\right)<\left(\begin{array}{c}
k+r-1 \\
r
\end{array}\right) \quad(r \in E N)
$$

where $\left.k=\# l_{f} \bigcup_{\varepsilon F}[f]\right),[f]$ is the conjugacy class of $f$.

PROOF. We show that if $[F]=\bigcup_{\mathrm{f} \in \mathrm{F}}[\mathrm{F}]$

$$
\#\left(F^{2}\right)<\#\left([F]^{2}\right)<\left(\begin{array}{c}
k+1 \\
2
\end{array}\right)
$$

Given $f, g \in F, f \neq g$, there is an element $g_{1}$ (say) in $[g] \subset$ [F] such that $\mathrm{fgf}^{-1}=\mathrm{g}_{1}$, and so $\mathrm{fg}=\mathrm{g}_{1} \mathrm{f}$.

Hence any 2-word in the elements of [F] consisting of two different letters is equal to another 2-word in the elements of $[F]$.

Thus $[F]^{2}$ has no more than $k$ elements $f^{2}$ and $k(k-1) / 2$ elements $f g$ where $f, g \in F, f \neq g$. It is clear that

$$
\#\left(F^{2}\right)<k+\frac{k(k-1)}{2}=\left(\begin{array}{c}
k+1 \\
2
\end{array}\right) \text {. }
$$

We suppose that the Theorem is true for any $r<n$, we show that it is also true for $r=n$.

We denote by $\phi(g)$ the number of all appearances of a $g \in[F]$ in the words of $\left([F]^{n}\right)^{\prime}$.

If all elements of [F] had the same chance to appear in ([F $])^{\prime}$ then for each $g \in[F], \phi(g)=\frac{n}{k}\left([F]^{n}\right)$.

Thus we may consider a $g \in[F]$ such that

$$
\#\left([F]^{n}\right)<\frac{k}{n} \phi(g)
$$

Since for any $f \in[F] \quad f \neq g$, there is some $f_{l}$ (say) such that $f_{g}=g f_{1}$. Hence without loss of generality we may assume that in any word of $\left([F]^{n}\right)$ ', either there is no $g$ or all $g^{\prime}$ 's keep the left place of the word. Now from each word of $\left([F]^{n}\right)^{\prime}$, where $g$ appears, cancel one $g$. The resulting $(n-1)$-words form a subset of distint elements of $[F]^{n-1}$; we denote this set by $g^{-1}\left([F]^{n}\right)^{\prime}$. Hence from our hypothesis it is clear that

$$
\phi(g)<\left(\begin{array}{c}
k+n-2 \\
n-1
\end{array}\right)+\phi_{1}(g)
$$

where $\phi_{1}(g)$ is the number of all appearances of $g$ in $g^{-1}\left([F]^{n}\right)^{\prime}$. Suppose that

$$
\phi_{1}(g)<\frac{n-1}{k}\left(\begin{array}{c}
k+n-2 \\
n-1
\end{array}\right)
$$

then by (3.1) and $(3.2)$ we obtain

$$
\#\left([F]^{n}\right)<\frac{k}{n}\left(1+\frac{n-1}{k}\right)\left(\begin{array}{c}
k+n-2 \\
n-1
\end{array}\right)<\left(\begin{array}{c}
k+n-1 \\
n
\end{array}\right)
$$

and so in this case there is nothing to show. If the inequality (3.3) does not occur, from (3.1) and (3.2) we havn 


$$
\left([F]^{n}\right)<\frac{k}{n}\left(\frac{k}{n-1}+1\right) \phi_{1}(g)<\frac{k(k+n-1)}{n(n-1)} \phi_{1}(g)
$$

In a similar way we define $\phi_{i}(g)(1<1<n-1)$ i.e. the number of appearances of $g$ in the collection $g^{-i}\left([F]^{n}\right)$ '.

As in (3.2)

$$
\left.\phi_{1-1}(g)<i_{n-1}^{k+n-1-1}\right)+\phi_{1}(g) \quad(i=2,3, \ldots, n-1)
$$

and if for some $i<n-1$

$$
\phi_{i}(g)<\frac{n-i}{k}\left(\begin{array}{c}
k+n-i-1 \\
n-i
\end{array}\right)
$$

it is nothing to show. If the inequality (3.4) does not occur for any $1<n-1$ we observe that

$$
\phi_{n-1}(g)<\frac{1}{k}\left(\begin{array}{l}
k \\
1
\end{array}\right)
$$

In this case we write

$$
\#\left([F]^{n}\right)<\frac{k(k+n-1) \ldots(k+1)}{n \cdot(n-1) \ldots(k+n-1) !}=\frac{(k-1) ! n !}{(k-1}
$$

and this completes the proof.

COROLLARY 3.1. If $G$ is abelian and $F$ is a finite subset of $G$ then,

$$
\left(F^{r}\right)<\left(\begin{array}{c}
\#+r-1 \\
r
\end{array}\right), \quad r \in N
$$

PROOF. Clear

LEMMA 3.1. Let $G$ be a discrete group with a normal subgroup $K$ such that $G / K$ is abelian, and let $\pi$ be the canonical map $\pi: G \rightarrow G / K$. If $F C G$ is such that \#F=\# $\pi(F)$, then the number of all $r$-words in the elements of $F(r \varepsilon N)$, in a given class of $G$ modulo $K$ can not be greater than

$$
\left(\begin{array}{l}
\left.\# \mathrm{~F}+\mathrm{r}^{-1}\right) \\
\mathbf{r}
\end{array}\right.
$$

PROOF. Let $\tilde{x} \in G / K$ fixed and $J_{r}=\pi^{-1}(\tilde{x}) \cap F^{r}$

Note that $J_{r}$ and $F^{r}$ in this proof mean collections of r-words, which as elements of $G$ may not be distinct.

We shall denote by \# $J_{r}$ the cardinality of $J_{r}$, and we shall show that

$$
\#\left([F]^{n}\right)<\left(\begin{array}{c}
\# F+1 \\
2
\end{array}\right)
$$

Let $x, y \in F$ and $x y \in J_{2}$, then $y x \in J_{2}$; in fact since $G / K$ is abelian $\pi(x y)=\pi(x) \pi(y)=\pi(y x)$. Now suppose that there is a $z \varepsilon F$ such that $x, z$ (or $z, x)$ is in $J_{2}$, i.e. $\pi(x, y)=\pi(x, z)$ and so $\pi(y)=\pi(z)$ and $\#(F)<\#$ F-contradiction. Hence it is clear that $\#_{2}<\# F$ and (3.5) follows.

We suppose that Lemma 3.1 is true for any $r>n-1$ and we show this for $r=n$. For 
fur some $\tilde{\mathrm{g}} \varepsilon \mathrm{G} / \mathrm{K}$, let

$$
J_{n}=\pi^{-1}(\tilde{g}) \cap F^{n}
$$

If all elements of $F$ had the same chance to appear in $J_{n}$, then the number of all appearances $\phi(x), x \in F$, of $x$ in the words of $J_{n}$ should be

$$
\phi(x)=\frac{n}{\# F} \#\left(U_{n}\right)
$$

We consider a $x \in F$ such that

$$
\# J_{n}<\frac{\# F}{n} \phi(x)
$$

From the set of all words in $J_{n}$ where $x$ has at least one entry we cancel one $x$. We denote by $x^{J} n$ the collection of all the resulting $(n-1)$-words. We show that $x_{n}^{J}$ is in a class of $G$ modulo $K$.

Let $w_{1}, w_{2}, w_{1}^{\prime}, w_{2}^{\prime}$, be words in the elements of $F$ such that $w_{1} \times w_{2}$, $w_{1}^{i} \times w_{2}^{\prime} \varepsilon J_{n^{*}}$ Since $\pi\left(w_{1} \times w_{2}\right)=\pi\left(w_{1}^{\prime} \times w_{2}^{\prime}\right)$, we have $\pi\left(w_{1} w_{2}\right)=\pi\left(w_{1}^{\prime} w_{2}^{\prime}\right)$ and $J_{n}$ is as we claimed.

Now, the set $\mathrm{x}_{\mathrm{n}}$ by our inductive hypothesis has cardinality no greater than $\left(\begin{array}{c}(\#+n-2 \\ n-1\end{array}\right)$ and so

$$
\phi(x)<\left(\begin{array}{c}
\# F+n-2 \\
n-1
\end{array}\right)+\phi_{1}(x)
$$

where $\phi_{1}(x)$ is the number of appearances of $x$ in $x^{J}$.

As in Proposition $(3.1),(3.7)$ in the case where

$$
\phi_{1}(x)<\frac{n-1}{\# F}\left(\begin{array}{c}
\#+n-2 \\
n-1
\end{array}\right)
$$

it is nothing to show. If the inequality above is not true by (3.6) and (3.7) we obtain

$$
\# J_{n}<\frac{\# F}{n} \frac{\# F+n-1}{n-1} \phi_{1}(x)
$$

We complete the proof in the same arguments as in Proposition 3.1 .

PROPOSITION 3.2. Any nilpotent group $G$ is an $A-g r o u p$ with $K(F)=\# F$ and

$\lambda=2 q-1$, where $q$ is the index of $G$ and $F$ is a finite set.

PROOF. Let $G=A_{0} \supset A_{1} \supset \ldots \supset A_{q-1} \supset A_{q}=\{e\}$ be the normal series of a nilpotent group $G$ of index $q$. We note that $A_{i-1} / A_{1}$ is the center of $G / A_{1}$

$(1<1<q-1)$ and we denote by $\pi_{1}$ the canonical map $G+G / A_{1}$.

It is obvious that for any $F \subset G$ and $r \in N$

$$
\#\left(F^{r}\right)<\#\left(\pi_{1}\left(F^{r}\right) \max \left\{\# \pi^{-1}(\tilde{g}) \cap F^{r}: \tilde{g} \in G / A_{1}\right\}\right. \text {. }
$$

We denote by $\left|\mathrm{F}^{\mathrm{r}}\right|_{1}$ the RHS of $(3.8)$.

We shall show that there are $q$ positive integers $m_{1}, m_{2}, \ldots, m_{q}$ such that 
$\#(F)=m_{1}+m_{2}+\ldots+m_{q}$ and

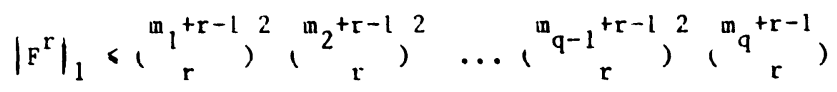

If $q=i$, i.e. $G$ is abelian. Corollary (3.1) implies (3.9). We suppose that (3.9) is true for each nilpotent gruup of index $q=p-1$.

Let $G$ be nilpotent of index p. Since $G / A_{1}$ is abellan by Lemma 3.1 if $\# \mathrm{~F}<\# \pi_{1}(\mathrm{~F})$.

We have, $\left.\quad\left|F^{r}\right|_{1} \leqslant 1^{\# F+r-1}\right)^{2}$.

Let $\#\left(\pi_{1}(F)\right)=m_{1}<\#(F)$, then $F$ can be written as

$$
F=\left\{x_{i} \alpha_{j}: 1<1<m_{1}, 1<j<\#(F)-m_{1}\right\}
$$

where $\pi_{1}\left(x_{i}\right) \neq \pi_{1}\left(x_{j}\right) \quad i \neq j, 1<j<m_{1}$, and all $\alpha_{j}$ 's are in $A_{1}$. By (3.8) we obtain,

$$
\left|F^{r}\right|_{1}<i^{m_{1}+r-1}, \# J_{1}
$$

where $J_{1}={ }_{1}^{-1}(g) F^{r}$, for some $g \in G / A_{1}$. Any element of $J_{1}$ can be written as

$$
x_{i_{1}} \alpha_{j_{1}} x_{i_{2}} \alpha_{j_{2}} \cdots x_{i_{r}}^{\alpha_{j}}
$$

where $\left(x_{i_{1}} x_{i_{2}} \ldots x_{i_{r}}\right) \in \tilde{g}$, each $i_{\leftarrow}(1<t<r)$ is one of $1,2, \ldots, m_{1}$ and each $j_{t}$ is one of $1,2, \ldots, \ldots, \mathrm{F}_{-} \mathrm{m}_{1}$.

By Lemma (3.1) the cardinality of all $x_{1} \ldots x_{1}$ in $\tilde{g}$ is $<\left(\begin{array}{c}m_{1}+r-1 \\ r\end{array}\right)$ and so

$$
\# J_{1}^{\prime}<\left(\begin{array}{c}
m_{1}^{+r-1} \\
r
\end{array}\right) \# J_{1}^{\prime}
$$

where

$$
J_{1}^{\prime}=x_{i_{1}} F_{1} x_{i_{2}} F_{1} \cdots x_{1} F_{1}
$$

$x_{1_{1}} x_{1_{2}} \cdots x_{i_{r}}$ is fixed suitably choosen from $(3.11)$ and belongs to $\tilde{g}$;

$$
F_{1}=\left\{\alpha_{j}: 1<j<\# F-m_{1}\right\}
$$

Note that if $q=2$, then $A_{1}$ is the center of $G$, all $\alpha_{j}$ 's commute with $x_{i}$ 's and by Corollary (3.1), $\left|F_{1}^{r}\right|<\left({ }_{r}^{F-m_{1}+r-1}\right)$; thus by (3.10) and $(3.12),(3.9)$ follows.

As in (3.8), for some $\tilde{g} \in G / A_{2}$ we have

$$
\# J_{1}^{\prime}<\# \pi_{2}\left(J_{1}^{\prime}\right) \#\left\{\pi_{2}^{-1}(\tilde{g}) \frown J_{1}^{\prime}\right\}
$$
obtain

Since $A_{1} / A_{2}$ is the center of $G / A_{2}$ and $F_{1} C A_{1}$, by $(3.10),(3.12)$ and (3.13) we

$$
\left|\mathrm{F}^{\mathrm{r}}\right|_{1}<\left(\mathrm{m}_{\mathrm{r}}^{+\mathrm{r}-1}\right)^{2} \cdot\left|\mathrm{F}_{1}^{\mathrm{r}}\right|_{1}
$$


where $\left|F_{1}^{r}\right|_{1}$ is defined as $\left|F^{r}\right|_{1}$.

Since $A_{1}$ is a nilpotent of index $p-1$ we apply our inductive hypothes is in (3.14) and for $q=p$ we obtain (3.9).

Now if we replace $m_{1}, \ldots, m_{q}$ in (3.9) by \#F we see that $G$ is an $A$ group with constants $k=1$ and $\lambda=2 q-1$.

PROPOSITION 3.3. The class of A-groups is closed under extensions by finite groups.

PROOF. We may write $G / A=\left\{d_{1} A, d_{2} A, \ldots, d_{s} A\right\}$ where $d_{1}, d_{2}, \ldots, d_{s}$ are $s$ representatives of all the different classes of $G / A$; without loss of generality let $d_{s}$ $=e$ the unit of $G$. We may also write

$$
d_{i} d_{j}=\alpha(i, j) d(i, j) \quad(1<i, j<s)
$$

where each $d(i, j)$ is one of $d_{1}, \ldots, d_{s}$ and each $a(i, j)$ is in $A$. $(1<t<m)$.

Let $F=\left\{d_{i_{1}} x_{1}, \ldots, d_{i_{m}} x_{m}\right\}, \# F=m$, each $d_{i_{t}}$ is one of $d_{1}, \ldots, d_{s}$ and $x_{t} \in A$

Let $\left\langle x_{i}\right\rangle=\left\{d_{j}^{-1} x_{i} d_{j}: j=1,2, \ldots, s\right\}(1<1<m)$ and

$$
d_{j_{1}} x_{i_{1}} d_{j_{2}} x_{i_{2}} \cdots d_{j_{r}} x_{i_{r}}
$$

be a typical r-word in the elements of F. Each word in (3.15) is in the set

$$
\begin{gathered}
\left\langle x_{i_{1}}\right\rangle \alpha\left(j_{1}, j_{2}\right) d\left(j_{1}, j_{2}\right) x_{i_{2}} \ldots d_{j_{r} x_{1_{r}}} \text { or in } \\
\left\langle x_{i_{1}}\right\rangle \alpha\left(j_{1}, j_{2}\right)\left\langle x_{i_{2}}\right\rangle \alpha\left(\cdot, j_{3}\right) \ldots d_{j_{r}} x_{1_{r}} \text { or finally in } \\
\left\langle x_{i_{1}}\right\rangle\left\langle x_{i_{2}}\right\rangle \ldots\left\langle x_{1_{r}}\right\rangle \text { a.d. }
\end{gathered}
$$

where a $\varepsilon A$ and $d \varepsilon\left\{d_{1}, d_{2}, \ldots, d_{s}\right\}$.

It is clear that the cardinality of the r-words in the elements of $F$, as in (3.15) is less than the candinality of the words in (3.16) in the elements of $\left\{\left\langle x_{1}\right\rangle, \ldots,\left\langle x_{m}\right\rangle\right\}$, which is a subset of the A-group, A. Thus $G$ inherits the growth of $\mathrm{A}$.

\section{REFERENCES}

1. PALMER, T. Classes of Non-Abe11an, Non-Compact, Locally Compact Groups, Rocky Moun. J. Math. 8(4) (1978), 683-741.

2. FOUNTAIN, J.B., RUMSAY, R.W. and WILliAmSON, J.H. "Functions of Measures on Compact Groups, Proc. Roy. Irish Acad. Sect. A., 76 (1976), 235-251.

3. KARANIKAS, C. and WILLIAMSON, J.H. Norms and Spectral for Certain Subalgebras of $M(G)$, Math. Proc. Camb. Phi1. Soc. 95 (1984), 109-122.

4. KARANIKAS, C. Wiener Pairs of Measure Algebras, Pac. Jour. Math. 139(1), (1989), 79-86. 


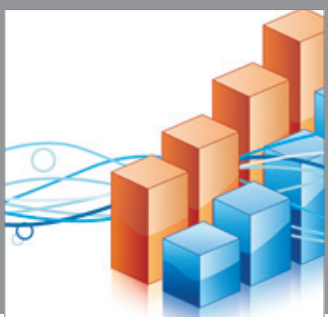

Advances in

Operations Research

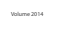

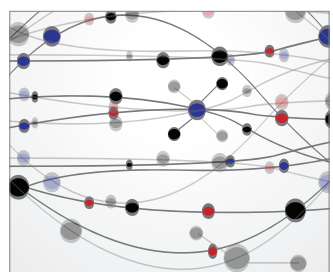

\section{The Scientific} World Journal
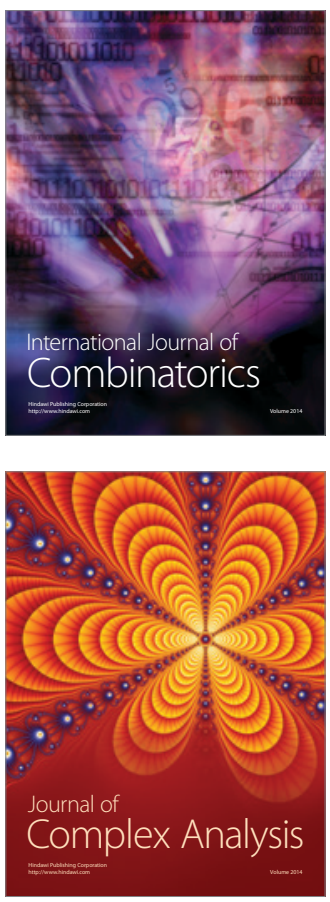

International Journal of

Mathematics and

Mathematical

Sciences
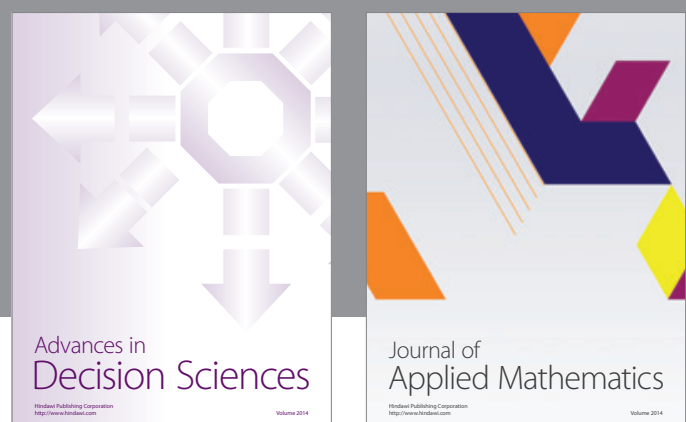

Journal of

Applied Mathematics
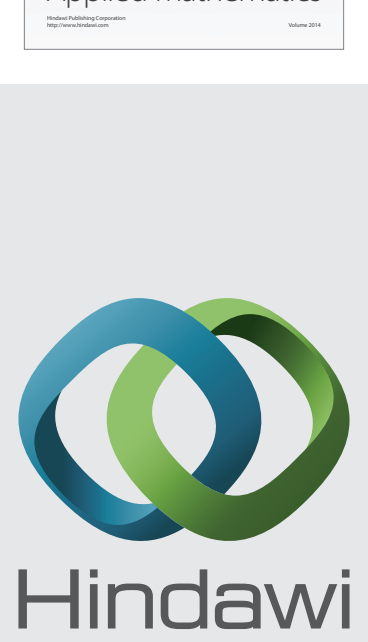

Submit your manuscripts at http://www.hindawi.com
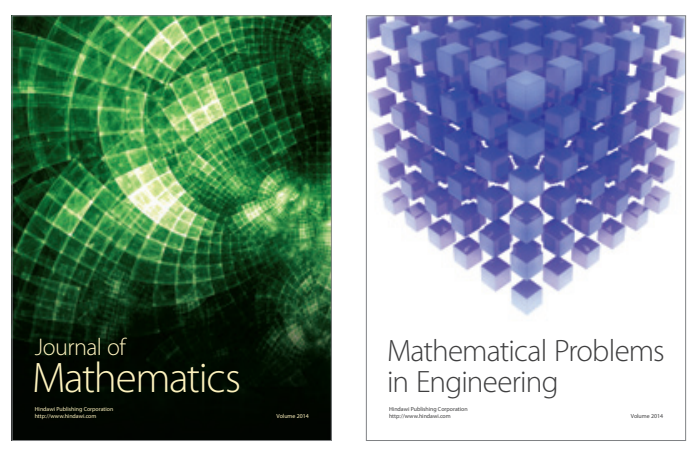

Mathematical Problems in Engineering
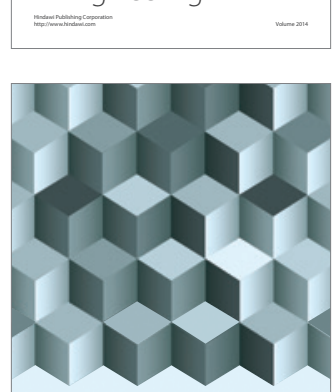

Journal of

Function Spaces
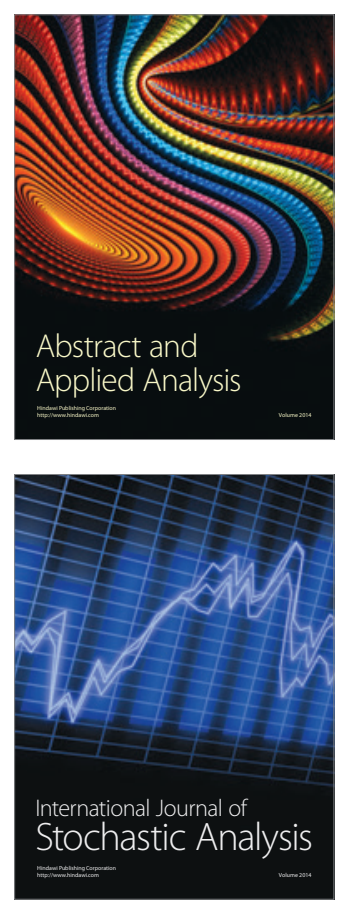

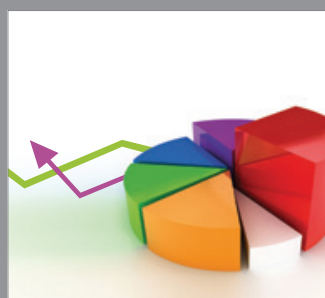

ournal of

Probability and Statistics

Promensencen
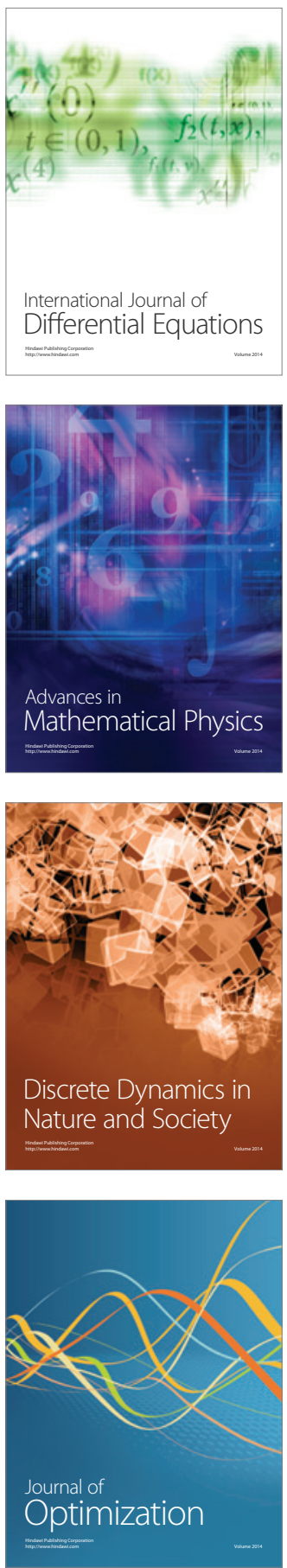